\title{
A Coding View of Network Recovery and Management
}

\author{
Tracey Ho and Muriel Médard ${ }^{1}$ \\ Laboratory for Information and Decision \\ Systems \\ Massachusetts Institute of Technology \\ Cambridge, MA 02139 \\ e-mail: $\{$ trace, medard $@$ mit.edu
}

\author{
Ralf Koetter \\ Coordinated Science Laboratory \\ University of Illinois \\ Urbana, IL 61801 \\ e-mail: koetter@csl.uiuc.edu
}

\begin{abstract}
A general description of the behavior of a communication network is given by codes specifying the relationships between each node's observable source processes and its input and output signals. In this work we focus on acyclic multicast networks, and consider two formulations for quantifying network management for link failure recovery, in terms of selecting between codes.
\end{abstract}

\section{INTRODUCTION}

Recent work has shown that coding (combining signals, not just routing and replication) is needed in some cases to achieve network capacity [1], and that linear coding is sufficient for multicast [2]. An algebraic framework introduced in [3] recovers these results and provides powerful analytical tools.

The full range of situations in which coding is needed for capacity is unclear. Apart from variations on the example in [2], our searches over several thousand randomly generated graphs have not yielded further examples.

Coding is often useful for robust link failure recovery, allowing for receiver-based recovery (i.e. only receiver nodes react under different failures) [3]. Different recovery strategies have different management overhead requirements. Describing network behavior as codes, we consider two formulations for quantifying essential management information by the number of codes needed across different failure scenarios. [4]

\section{MODEL}

We consider a delay-free acyclic network with unit capacity directed links, and one or more source nodes, at which one or more discrete, independent, unit entropy rate random processes are observable. The multicast connection problem is to transmit all processes through the network to one or more receiver nodes, each distinct from the source nodes. ${ }^{2}$ We call an incoming incident link of a receiver node a terminal link.

A code for a node is a function specifying its outputs (i.e. the signal on each of its outgoing links, and, for receiver nodes, its output processes) from signals on its incoming links and processes observable at that node. In a linear code, these functions are all linear. A centralized code is a set of codes for all nodes in the network, considered valid if all receiver nodes can reconstruct the input signals and output them in order.

III. MANAGEMEnT FOR Link Failure Recovery We assume that the zero signal is observed on failed links. We compare receiver-based recovery and network-wide recovery (i.e. any node may react to failures) using linear codes. For the multicast case where we consider terminal link failures, we also analyze receiver-based recovery where signals on links remain linear functions of input signals and source processes,

${ }^{1}$ This work was supported by NSF CAREER grant 0093349.

${ }^{2}$ Our model admits multiple source nodes and parallel links. but outputs at the receivers may be nonlinear functions of their input signals.

In a centralized formulation, the management requirement is given in terms of the number $n_{c}$ of centralized codes, by $\left\lceil\log _{2} n_{c}\right\rceil$ bits.

Theorem 1 For a single-receiver network with $r$ processes and a minimum cut capacity of $c$, tight bounds ${ }^{3}$ on the number of codes needed for the no-failure scenario and all single link failures, assuming they are recoverable, are:

\begin{tabular}{|c|c|c|}
\hline $\begin{array}{c}\text { recovery } \\
\text { scheme }\end{array}$ & $\begin{array}{l}\text { lower } \\
\text { bound }\end{array}$ & $\begin{array}{l}\text { upper } \\
\text { bound }\end{array}$ \\
\hline $\begin{array}{c}\text { linear } \\
\text { receiver- } \\
\text { based }\end{array}$ & $\left\lceil\frac{c}{c-r}\right\rceil$ & $\begin{cases}r+1 & \text { for } r=1 \text { or } c-1 \\
r & \text { for } 2 \leq r \leq c-2\end{cases}$ \\
\hline $\begin{array}{c}\text { linear } \\
\text { network- } \\
\text { wide }\end{array}$ & $\left\lceil\frac{c+1}{c-r+1}\right\rceil$ & $\begin{cases}2 & \text { for } r=1 \\
r & \text { for } r=2,3, c-1 \\
r-1 & \text { for } 4 \leq r \leq c-2\end{cases}$ \\
\hline
\end{tabular}

Theorem 2 For a multicast problem with $r$ processes and two or more receivers, let each receiver $\beta$ have $d_{\beta}$ terminal links, and let $d_{\min }=\min _{\beta} d_{\beta}$. Tight bounds ${ }^{3}$ on the number of codes needed for the no-failure case and failures of individual terminal links, assuming they are recoverable, are:

\begin{tabular}{|c|c|c|}
\hline $\begin{array}{c}\text { recovery } \\
\text { scheme }\end{array}$ & $\begin{array}{c}\text { lower } \\
\text { bound }\end{array}$ & $\begin{array}{c}\text { upper } \\
\text { bound }\end{array}$ \\
\hline $\begin{array}{c}\text { linear } \\
\text { receiver- } \\
\text { based }\end{array}$ & $\left\lceil\frac{d_{\min }}{d_{\min }-r}\right]$ & $\left\{\begin{array}{c}r+1 \text { for } r=1 \text { or } d_{\min }-1 \\
\text { for } 2 \leq r \leq d_{\min }-2\end{array}\right.$ \\
\hline $\begin{array}{c}\text { linear } \\
\text { network- } \\
\text { wide }\end{array}$ & $\left\{\left[\begin{array}{c}\frac{d_{\min }+1}{d_{\min }-r+1}+\frac{d_{\min }+1}{d_{\min }-r+1}-1 \text { if }\left[\frac{d_{\min }+1}{d_{\min }-r+1}\right] \\
\text { otherwise }\end{array}\right.\right.$ & $\left\{\begin{array}{c}r+1 \text { for } r=1 \text { or } d_{\min }-1 \\
\text { for } 2 \leq r \leq d_{\min }-2\end{array}\right.$ \\
\hline $\begin{array}{c}\text { nonlinear } \\
\text { receiver } \\
\text { based }\end{array}$ & $\left\{\begin{array}{l}r \text { for } 1 \leq r=d_{\min }-1 \\
1 \text { for } r=1 \text { or } r \leq d_{\min }-2\end{array}\right.$ & $r$ \\
\hline
\end{tabular}

A node-based formulation takes the sum over all nodes of the $\log$ of the number of different behaviors for each node.

Theorem 3 For the single-receiver case with linear codes, the minimum node-based management requirement for terminal link failures and the no-failure scenario is achieved with receiver-based schemes.

\section{REFERENCES}

[1] R. Ahlswede, N. Cai, S.-Y.R. Li and R.W. Yeung, "Network Information Flow", IEEE-IT, vol. 46, pp. 1204-1216, 2000.

[2] S.-Y.R. Li and R.W. Yeung, "Linear Network Coding", preprint, 1999.

[3] R. Koetter and M. Médard, "An Algebraic Approach to Network Coding", Proceedings of the 2001 IEEE International Symposium on Information Theory, pp. 104, 2001.

[4] T. Ho, M. Médard and R. Koetter, "A Coding View of Network Recovery and Management for Single-Receiver Communications", Proceedings of the 2002 Conference on Information Sciences and Systems, 2002.

\footnotetext{
${ }^{3}$ for any values of $r, c$ and $d_{\min }$, there are examples for which
} the bounds are met with equality 